\title{
Fast gain and phase recovery of semiconductor optical amplifiers based on submonolayer quantum dots
}

Cite as: Appl. Phys. Lett. 107, 201102 (2015); https://doi.org/10.1063/1.4935792

Submitted: 20 July 2015 • Accepted: 03 November 2015 • Published Online: 16 November 2015

Bastian Herzog, Nina Owschimikow, Jan-Hindrik Schulze, et al.
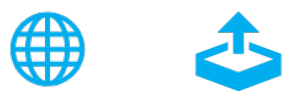

\section{ARTICLES YOU MAY BE INTERESTED IN}

Strong amplitude-phase coupling in submonolayer quantum dots

Applied Physics Letters 109, 201102 (2016); https://doi.org/10.1063/1.4967833

Atomic structure and optical properties of InAs submonolayer depositions in GaAs

Journal of Vacuum Science \& Technology B 29, $04 D 104$ (2011); https://

doi.org/10.1116/1.3602470

High-power semiconductor disk laser based on InAs / GaAs submonolayer quantum dots

Applied Physics Letters 92, 101123 (2008); https://doi.org/10.1063/1.2898165

田QBLOX

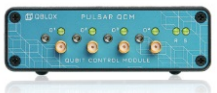

1 qubit
Shorten Setup Time

Auto-Calibration

More Qubits

Fully-integrated

Quantum Control Stacks

Ultrastable DC to $18.5 \mathrm{GHz}$

Synchronized $<<1$ ns

Ultralow noise

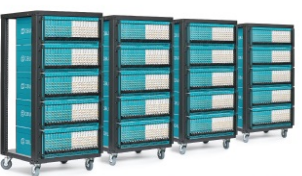

100s qubits

visit our website > 


\title{
Fast gain and phase recovery of semiconductor optical amplifiers based on submonolayer quantum dots
}

\author{
Bastian Herzog, ${ }^{1, a)}$ Nina Owschimikow, ${ }^{1}$ Jan-Hindrik Schulze, ${ }^{2}$ Ricardo Rosales, ${ }^{2}$ \\ Yücel Kaptan, ${ }^{7}$ Mirco Kolarczik, ${ }^{1}$ Thomas Switaiski, ${ }^{1}$ André Strittmatter, ${ }^{2}$ Dieter Bimberg, ${ }^{2}$ \\ Udo W. Pohl, ${ }^{2}$ and Ulrike Woggon ${ }^{1}$ \\ ${ }^{1}$ Institut für Optik und Atomare Physik, Technische Universität Berlin, Straße des 17. Juni 135, 10623 Berlin, \\ Germany \\ ${ }^{2}$ Institut für Festkörperphysik, Technische Universität Berlin, Hardenbergstrasse 36, 10623 Berlin, Germany
}

(Received 20 July 2015; accepted 3 November 2015; published online 16 November 2015)

\begin{abstract}
Submonolayer quantum dots as active medium in opto-electronic devices promise to combine the high density of states of quantum wells with the fast recovery dynamics of self-assembled quantum dots. We investigate the gain and phase recovery dynamics of a semiconductor optical amplifier based on InAs submonolayer quantum dots in the regime of linear operation by one- and two-color heterodyne pump-probe spectroscopy. We find an as fast recovery dynamics as for quantum dot-ina-well structures, reaching 2 ps at moderate injection currents. The effective quantum well embedding the submonolayer quantum dots acts as a fast and efficient carrier reservoir. (C) 2015 AIP Publishing LLC. [http://dx.doi.org/10.1063/1.4935792]
\end{abstract}

Quantum-confined epitaxial semiconductor systems such as two-dimensional (2D) quantum well (QW) layers or zero-dimensional (OD) quantum dot (QD) ensembles have a wide range of applications from solar cells to telecommunications. Although QWs offer a high density of states (DOS), narrow spectral emission linewidth, and thus a high modal gain, the strong localization created by OD confinement in QDs leads to very fast gain dynamics and low temperaturestable lasing thresholds, ${ }^{1-5}$ however, at the expense of a reduced DOS. Naturally, it would be highly desirable to design a gain medium that combines the speed of QDs with the high DOS and spectral homogeneity of QWs.

An approach to obtain a high areal density of OD localization centers is the growth of submonolayer (SML) islands. In this process, InAs is deposited on GaAs in quantities of less than a monolayer (ML), and overgrown with a thin GaAs layer, on which again a submonolayer of InAs is grown. ${ }^{6,7}$ The strain induced by the lattice mismatch of both compound semiconductors leads to a vertical alignment of the InAs SML depositions during the growth process, thereby forming InAs-rich islands acting as localization centers inside an effective InGaAs QW with low In content. The emission wavelength of these structures, referred to here as SML QDs, is typically between 900 and $1000 \mathrm{~nm}$, but the incorporation of antimony allows to push it into the O-band telecommunications wavelength range. ${ }^{8}$ The areal density of the SML QDs is about $10^{12} \mathrm{~cm}^{-2}$, and thus it is an order of magnitude larger than for self-assembled Stranski Krastanow (SK) QDs. ${ }^{9}$ Investigations of unprocessed SML structures have revealed both $0 \mathrm{D}$ and $2 \mathrm{D}$ features in spectra and dynamics, ${ }^{10-13}$ caused by the large exciton Bohr radius, which averages over many islands. ${ }^{8}$

The large areal density of SML QDs, a typically narrower emission spectrum, and stronger interaction with p-polarized light than for SK QDs are promising for the

a)BHerzog@physik.tu-berlin.de application of SML QDs as active medium in opto-electronic devices. ${ }^{14}$ Examples include edge-emitting lasers ${ }^{15}$ and vertical-cavity surface-emitting lasers, ${ }^{16-19}$ as well as photodetectors and solar cells. ${ }^{20,21}$ The comparatively high DOS is reflected in a maximum modal gain reported for InAs SML QDs of $44 \mathrm{~cm}^{-1}$, compared to $33 \mathrm{~cm}^{-1}$ per layer for SK QDs at $980 \mathrm{~nm} .{ }^{15,22,23}$ An equally favorable gain recovery dynamics as in SK QDs still has to be verified.

In this letter, we investigate the gain and phase recovery dynamics of a semiconductor optical amplifier (SOA) based on the InAs SML QDs. SOAs are applied not only for fast amplification but also for fast (cross) gain and phase modulation. To extract the relevant gain and phase recovery times for amplification, we apply one-color pump-probe spectroscopy (1cPP) at the spectral electroluminescence (EL) maximum, and also two-color pump-probe spectroscopy (2cPP) to quantify recovery times for cross gain modulation applications. For our investigations, we use SOAs containing five layers of InAs/GaAs SML stacks in the active region, with the emission centered at $965 \mathrm{~nm}(1.28 \mathrm{eV}$, see Fig. 1(a)). The $\mathrm{p}$-i-n device structure was grown on a Si-doped GaAs substrate using metal-organic vapour phase epitaxy. Each SML stack consists of a sixfold deposition of nominally $0.4 \mathrm{ML}$ InAs and 1.6 ML GaAs (see inset of Fig. 1(b)), the stacks are separated by a $40 \mathrm{ML}$ GaAs spacer. Lateral waveguiding is achieved by a ridge of $2 \mu \mathrm{m}$ width shallow-etched down to about $80 \mathrm{~nm}$ above the active medium. The facets of the device are tilted $8^{\circ}$ against the normal to suppress optical feedback. The inset of Figure 1(a) shows the SOA strip and the contact probe used for current supply. For thermal stabilization, the device was mounted onto a water-cooled heat sink held at $13^{\circ} \mathrm{C}$.

EL spectra of the device are shown in Fig. 1(a). For high injection current, the SML transition shows a Gaussian shape with a full width at half maximum (FWHM) of $25 \mathrm{meV}$. Additionally, we find unstructured pronounced wings on the blue and on the red sides of the emission, which we attribute 


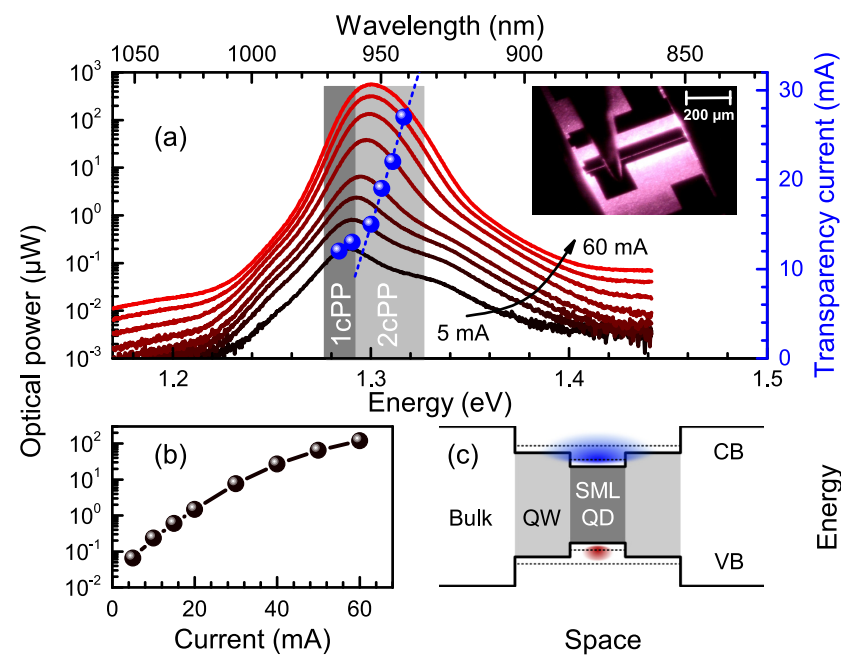

FIG. 1. (a) Electroluminescence spectra of the SML QD SOA for 5, 10, 15, $20,30,40,50$, and $60 \mathrm{~mA}$ injection current. The dark gray and light gray windows illustrate the spectral range of the one- and two-color pump-probe experiments, respectively. The blue dots mark the transparency current, extracted from two-color pump probe measurements, the dashed line illustrates the linear increase at wavelengths below $960 \mathrm{~nm}$. Inset: Image of the device in operation. (b) Spectrally integrated emission as a measure for the light-current characteristics. (c) Energy scheme and schematic carrier wavefunctions of the SML QDs, CB: conduction band and VB: valence band.

to Boltzmann and Urbach tails, respectively. ${ }^{24}$ The emission peak shifts from $965 \mathrm{~nm}$ to $955 \mathrm{~nm}$ with increasing current due to state filling. The light-current characteristics are shown in Fig. 1(b). To prevent device degradation, the injection current was limited to $60 \mathrm{~mA}$ where the SOA is still operating in non-saturation regime, which indicates a high DOS of the SML stacks. The maximum applied current corresponds to a nominal current density of $6 \mathrm{kA} / \mathrm{cm}^{2}$. Injection currents rather than current densities are quoted, since current spreading-which is significant in shallow etched QW devices $^{25}$ — was not quantified for our SML devices.

All pump-probe measurements were performed in the linear operating mode of the SOA with pulse powers set to $30 \mu \mathrm{W}$ and $300 \mu \mathrm{W}$ for probe and pump pulse, corresponding to $0.4 \mathrm{pJ} / \mathrm{pulse}$ and $4 \mathrm{pJ} / \mathrm{pulse}$, respectively. The relevant wavelengths for the $1 \mathrm{cPP}$ and $2 \mathrm{cPP}$ measurements are marked in Figure 1(a) as a dark (1cPP) and light gray (2cPP) area, respectively.

A scheme of the pump-probe setup is shown in Fig. 2. Pump and probe pulses are generated by a Toptica FemtoFiber pro laser system, in which a mode-locked Er-doped fiber oscillator seeds two amplifiers followed by highly nonlinear fibers to obtain independently tunable supercontinua. From these supercontinua, suitable parts are selected by an amplitude mask in the Fourier plane of a $4 \mathrm{f}$ pulse shaper. Typically, the pulses have a temporal and spectral FWHM of $250 \mathrm{fs}$ and $10 \mathrm{~nm}$ (13 meV), respectively. The co-polarized pump and probe pulses are colinearly coupled into the input facet of the SOA. Discrimination between pump and probe pulses is achieved by heterodyne detection, in which the probe pulse is divided into a probe and a reference part prior to coupling into the device. The probe part is slightly frequency-shifted by an acousto-optical modulator (AOM), yielding a temporal beating when interfering with the reference pulse on two balanced detectors. Amplitude

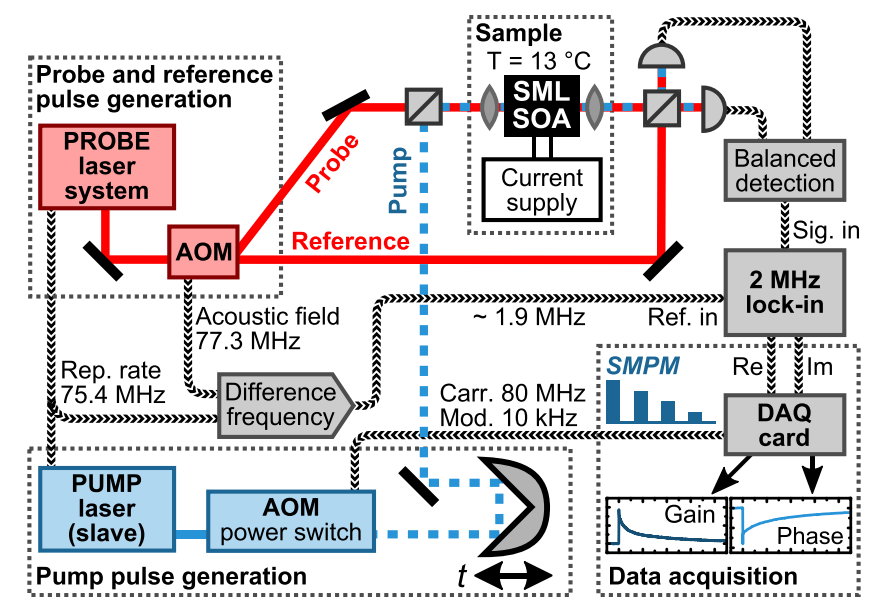

FIG. 2. Schematic of the heterodyne pump-probe experiment. AOM: acousto-optical modulator, DAQ: data acquisition, and SMPM: simultaneous multi-power measurement.

and phase of the beating signal are detected by a fast lock-in detector (Perkin Elmer DSP7280). From the transmitted probe amplitude, we derive the differential gain $\Delta G$, defined according to $\Delta G=10 \times \log V / V_{0}$, where $V$ and $V_{0}$ are the lock-in voltage in the presence and in the absence of the pump pulse. An AOM in the pump pulse path allows us to simultaneously record data at several pump power settings, to rule out nonlinear effects on the measurements. ${ }^{26}$ Note that a positive $\Delta G$ is equivalent to an amplification of the probe pulse as a result of excess carriers generated by the absorbed pump pulse. Outside the temporal pump-probe overlap, the cases of $\Delta G(t)>0$ and $\Delta G(t)<0$ will thus be named absorption and amplification regime (always with respect to the pump pulse), while transparency occurs at the transition $\Delta G(t)=0$. The dependence of the transparency current $I_{t r}$ on the wavelength of the pump pulse in $2 \mathrm{cPP}$ experiments with the probe at $980 \mathrm{~nm}$ is shown in Fig. 1(a) as blue dots. $I_{t r}$ roughly samples the number of energy states up to the pump energy window. We observe a steep and linear increase for pump pulse wavelengths shorter than the EL peak at $960 \mathrm{~nm}$ indicating a constant (QW-like) DOS, while for longer wavelengths the reduced slope hints at a lower DOS.

The differential gain and phase changes measured in $1 \mathrm{cPP}$ experiments with pump and probe pulses centered at the spectral maximum of the device emission at $965 \mathrm{~nm}$ are shown in Figs. 3(a) and 3(b), respectively, for currents between $10 \mathrm{~mA}$ and $60 \mathrm{~mA}$ as filled symbols. At low injection current, we observe optical pumping, reflected by a positive $\Delta G$ at the probe wavelength. Between $10 \mathrm{~mA}$ and $20 \mathrm{~mA}$ injection current, the gain recovery curve changes its sign from absorbing to amplifying with a negative $\Delta G$. The transparency current $I_{t r}$ in this wavelength range is about $12 \mathrm{~mA}$. Common to all curves is an initially fast gain recovery, followed by a slow return to equilibrium.

The gain recovery traces can be decomposed into three distinct parts. An immediate response at the sub-ps timescale is assigned to a combination of coherent interaction and ultrafast carrier heating (see inset in Fig. 3(a)). For this process, we do not extract a time constant, as in our experiment the shape of the probe pulse is nonlinearly modified at short 

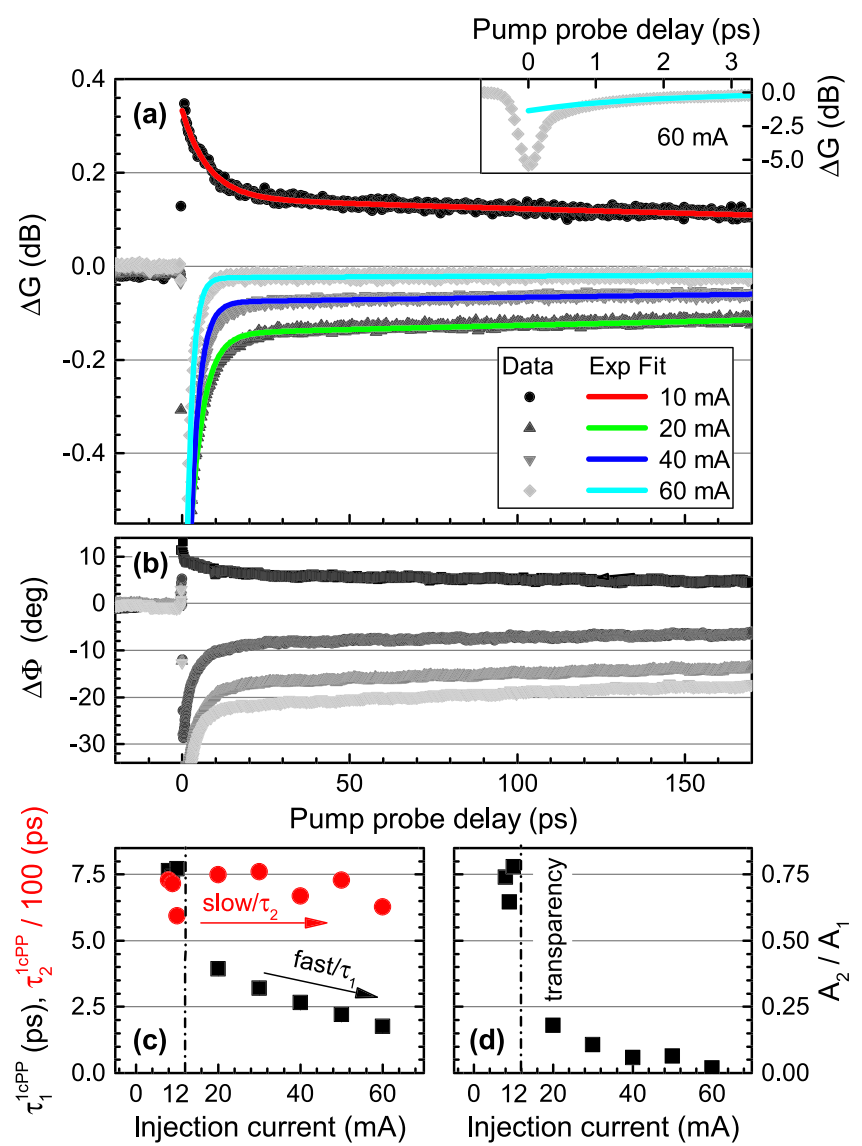

FIG. 3. Gain (a) and phase (b) recovery dynamics measured in one-color pump-probe experiments at $965 \mathrm{~nm}$ for injection currents from $10 \mathrm{~mA}$ to $60 \mathrm{~mA}$. The gray symbols represent data, and solid lines denote the exponential fit. (c) Time constants extracted from two-exponential fits of the gain recovery. (d) Amplitude ratio $A_{2} / A_{1}$.

delay times causing an additional contribution to $\Delta G$. This ultrafast feature is followed by a recovery characterized by a few ps time constant, which reflects the re-filling of the depleted states by other carriers. The long-time recovery belongs to the recovery of the equilibrium carrier density of the system by electrical injection. ${ }^{26}$

For times longer than $1 \mathrm{ps}$, the gain recovery traces are well described by a sum of exponential functions $\Delta G(t)$ $=A_{1} \exp \left(-t / \tau_{1}\right)+A_{2} \exp \left(-t / \tau_{2}\right)$, with the two time constants differing by two orders of magnitude. The dependence of the retrieved time constants on the injection current is displayed in Fig. 3(c). The fast time constant $\tau_{1}^{1 c P P}$ is in the range of 6-8 ps for low currents, and decreases to about 2 ps for high currents. QW-based devices have a somewhat slower carrier recovery time of the order of $10 \mathrm{ps,} \mathrm{as} \mathrm{the} \mathrm{res-}$ ervoir in this case is mainly located within the surrounding barrier material and the recovery includes a spatial diffusion step. ${ }^{27,28}$ Electronic states of SK QDs of moderate areal densities, in particular, in QD-in-a-well (DWELL) structures, are much more efficiently filled with carriers accumulated at higher energies in either QD excited states or states of the embedding QW, which leads to very fast re-equilibration of the gain with time constants of few ps. ${ }^{29,30}$ The fast recovery we observe for SML QDs shows that the potential landscape formed by other SML islands and the effective QW provides an efficient carrier reservoir with fast down-scattering

channels. The energetic structure of the SML QDs is illustrated in Fig. 1(c). 8-band $\mathrm{k}^{*} \mathrm{p}$ calculations have shown a high degree of localization for the hole wavefunction, while the electron wavefunction extends to neighboring SML islands and the embedding (energetically close) QW. ${ }^{8}$ The carriers localized in the active region are thus likely to respond fast to changes in the potential caused by the creation or annihilation of an exciton.

The second time constant $\tau_{2}^{1 c P P}$ for the SML SOA is about $600-800$ ps. The dependence of its magnitude on the injection current is weak, in contrast to the contribution to the total amplitude, which decreases down to below $5 \%$ for high currents (Fig. 3(d)). This is promising for telecommunication applications as it allows for pattern-effect-free data transmission. ${ }^{31,32}$ The value of 800 ps for $\tau_{2}^{1 c P P}$ in the absorption regime agrees well with the radiative lifetime reported for SML excitons. ${ }^{33,34}$

The dynamics observed for the phase recovery is similar to the gain recovery, except for the amplitude distribution between fast and slow components (Fig. 3(b)). The phase samples a broader spectral range than the gain, thus the amplitude of the fast component is not as pronounced. Additionally, the SML agglomerations together with the effective QW form a continuous spectral background, which leads to a refractive index change at the probe energy larger than for the relatively well separated QW of SK-DWELL devices.

Results of two-color pump-probe experiments with the probe pulse wavelength fixed to the maximum of the device emission at $960 \mathrm{~nm}$ and the pump pulse energy covering a window from $960 \mathrm{~nm}$ to $940 \mathrm{~nm}$ central wavelength on the blue shoulder of emission peak are shown in Figs. 4(a)-4(c) for the absorption, transparency, and amplification regime, respectively. In Figs. 4(a) and 4(c), the curves are normalized to their maximum to visualize the different weights of fast and slow relaxation components. We observe no systematic variation of the absolute amplitudes of $\Delta G$, as it would

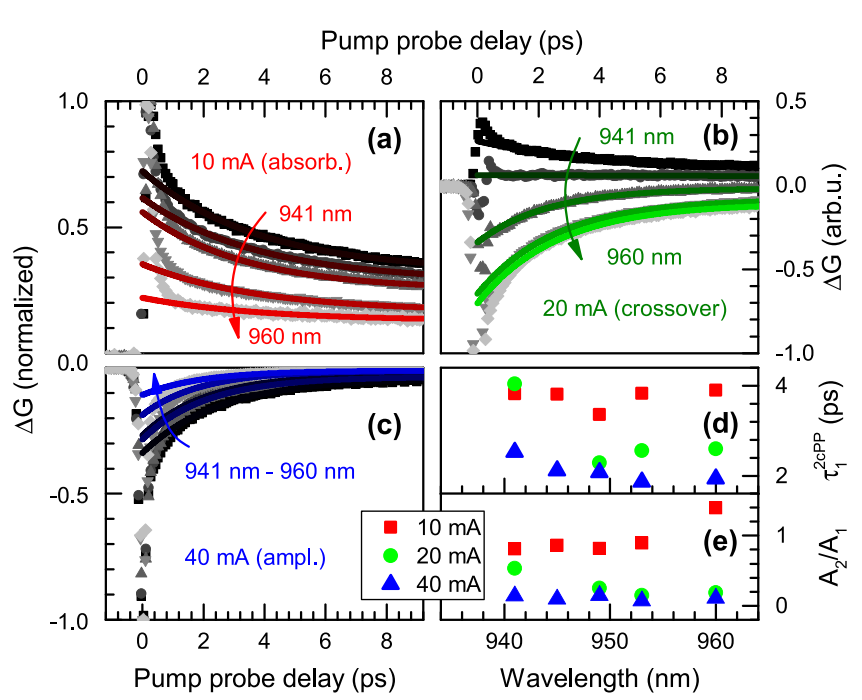

FIG. 4. Gain recovery in two-color pump-probe experiments with probe pulse at $960 \mathrm{~nm}$ and pump at 960, 953,949, 945, and $941 \mathrm{~nm}$. (a) Absorbing regime at $10 \mathrm{~mA}$, (b) crossover from amplifying to absorbing regime at $20 \mathrm{~mA}$, and (c) amplifying regime at $40 \mathrm{~mA}$ injection current. (d) Time constants $\tau_{1}^{2 c P P}$ and (e) amplitude ratios $\mathrm{A}_{2} / \mathrm{A}_{1}$. 
be expected for a structured spectrum with excited SML QD states at higher energy. ${ }^{35}$ As the slow component is identical for one- and two-color experiments, we show only the first ten picoseconds of the traces. The fitted recovery time constants $\tau_{1}^{2 c P P}$ are displayed in Fig. 4(d) and show a general decrease with increasing injection current. A slight slowing down for shorter pump wavelengths occurs as the fast spectral hole burning or carrier heating contributions induced by the pump shift out of the probe window. In the amplifying regime at $40 \mathrm{~mA}$ injection current (Fig. 4(c)), interesting for possible cross gain modulation, we observe a $\tau_{1}^{2 c P P}$ of $2.5-1.9 \mathrm{ps}$, with the fastest rate for the resonant experiment at $960 \mathrm{~nm}$. The relative amplitude of the slow component is shown in Fig. 4(e). The gain recovery for the crossover regime around the (wavelength-dependent) transparency current at $20 \mathrm{~mA}$ and the absorbing regime at $10 \mathrm{~mA}$ are shown in Figs. 4(a) and 4(b), respectively. Also for these currents, the initial recovery is fast (Fig. 4(d)); however, the long-term component gains weight increasingly and amounts to more than a half of the total amplitude in the absorbing case.

In conclusion, we investigated the gain and phase recovery dynamics of an SOA based on InAs SML QDs in a GaAs matrix as an active medium by one- and two-color heterodyne pump-probe spectroscopy. The recovery of gain and phase has been found to proceed in two stages. The first stage is described by a time constant $\tau_{1}$ of few ps, which is as fast as the recovery times observed for devices based on SK QDs. We attribute this to the effective InGaAs QW embedding the SML QDs acting as an efficient carrier reservoir. The second stage is an overall re-equilibration of the system and proceeds with a time constant $\tau_{2}$ of about $700 \mathrm{ps.}$ The high relative amplitude of the fast component, in particular, at high injection current, is promising for the application of SML QD-based devices in telecommunications as amplifiers and modulators.

We are grateful to Holger Schmeckebier for the help with device preparation. This research was funded by Deutsche Forschungsgemeinschaft via Sfb 787 and GRK 1558.

${ }^{1}$ Quantum Well Lasers, edited by J. P. S. Zory (Academic Press, 1993).

${ }^{2}$ A. A. Ukhanov, A. Stintz, P. G. Eliseev, and K. J. Malloy, Appl. Phys. Lett. 84, 1058 (2004).

${ }^{3}$ S. Dommers, V. V. Temnov, U. Woggon, J. Gomis, J. Martinez-Pastor, M. Laemmlin, and D. Bimberg, Appl. Phys. Lett. 90, 033508 (2007).

${ }^{4}$ J. Gomis-Bresco, S. Dommers, V. Temnov, U. Woggon, M. Laemmlin, D. Bimberg, E. Malic, M. Richter, E. Schöll, and A. Knorr, Phys. Rev. Lett. 101, 256803 (2008).

${ }^{5}$ T. Akiyama, H. Kuwatsuka, T. Simoyama, Y. Nakata, K. Mukai, M. Sugawara, O. Wada, and H. Ishikawa, IEEE J. Quantum Electron. 37, 1059-1065 (2001).

${ }^{6}$ S. Krishna, D. Zhu, J. Xu, K. K. Linder, O. Qasaimeh, P. Bhattacharya, and D. L. Huffaker, J. Appl. Phys. 86, 6135 (1999).

${ }^{7}$ T. Niermann, F. Kießling, M. Lehmann, J.-H. Schulze, T. D. Germann, K. Pötschke, A. Strittmatter, and U. W. Pohl, J. Appl. Phys. 112, 083505 (2012).
${ }^{8}$ D. Quandt, J.-H. Schulze, A. Schliwa, Z. Diemer, C. Prohl, A. Lenz, H. Eisele, A. Strittmatter, U. W. Pohl, M. Gschrey, S. Rodt, S. Reitzenstein, D. Bimberg, M. Lehmann, and M. Weyland, Phys. Rev. B 91, 235418 (2015).

${ }^{9}$ A. Lenz, H. Eisele, J. Becker, J.-H. Schulze, T. D. Germann, F. Luckert, K. Pötschke, E. Lenz, L. Ivanova, A. Strittmatter, D. Bimberg, U. W. Pohl, and M. Dähne, J. Vacuum Sci. Technol. B 29, 04D104 (2011).

${ }^{10}$ Z. C. Xu, K. Leosson, D. Birkedal, V. Lyssenko, J. M. Hvam, and J. Sadowski, Nanotechnology 14, 1259 (2003).

${ }^{11}$ L. C. Andreani, G. Panzarini, and J.-M. Gérard, Phys. Rev. B 60, 13276 (1999).

${ }^{12}$ Z. C. Xu, Y. T. Zhang, J. M. Hvam, J. J. Xu, X. S. Chen, and W. Lu, Appl. Phys. Lett. 89, 013113 (2006).

${ }^{13}$ A. Manohar, S. Sengupta, H. Ghadi, and S. Chakrabarti, J. Lumin. 158, 149 (2015).

${ }^{14}$ J. O. Kim, S. Sengupta, A. V. Barve, Y. D. Sharma, S. Adhikary, S. J. Lee, S. K. Noh, M. S. Allen, J. W. Allen, S. Chakrabarti, and S. Krishna, Appl. Phys. Lett. 102, 011131 (2013).

${ }^{15}$ D. Arsenijevic, C. Y. Liu, A. Payusov, M. Stubenrauch, and D. Bimberg, IEEE Photonics Technol. Lett. 24, 906 (2012).

${ }^{16}$ F. Hopfer, A. Mutig, M. Kuntz, G. Fiol, D. Bimberg, N. N. Ledentsov, V. A. Shchukin, S. S. Mikhrin, D. L. Livshits, I. L. Krestnikov, A. R. Kovsh, N. D. Zakharov, and P. Werner, Appl. Phys. Lett. 89, 141106 (2006).

${ }^{17}$ S. A. Blokhin, N. A. Maleev, A. G. Kuzmenkov, A. V. Sakharov, M. M. Kulagina, Y. M. Shernyakov, I. I. Novikov, M. V. Maximov, V. M. Ustinov, A. R. Kovsh, S. S. Mikhrin, N. N. Ledentsov, G. Lin, and J. Y. Chi, IEEE J. Quantum Electron. 42, 851 (2006).

${ }^{18}$ A. G. Kuzmenkov, V. M. Ustinov, G. S. Sokolovskii, N. A. Maleev, S. A. Blokhin, A. G. Deryagin, S. V. Chumak, A. S. Shulenkov, S. S. Mikhrin, A. R. Kovsh, A. D. McRobbie, W. Sibbett, M. A. Cataluna, and E. U. Rafailov, Appl. Phys. Lett. 91, 121106 (2007).

${ }^{19}$ N. N. Ledentsov, D. Bimberg, F. Hopfer, A. Mutig, V. A. Shchukin, A. Savel'ev, G. Fiol, E. Stock, H. Eisele, M. Dähne, D. Gerthsen, U. Fischer, D. Litvinov, A. Rosenauer, S. S. Mikhrin, A. R. Kovsh, N. D. Zakharov, and P. Werner, Nanoscale Res. Lett. 2,417 (2007).

${ }^{20}$ D. Z.-Y. Ting, S. V. Bandara, S. D. Gunapala, J. M. Mumolo, S. A. Keo, C. J. Hill, J. K. Liu, E. R. Blazejewski, B. Rafol, and Y.-C. Chang, Appl. Phys. Lett. 94, 111107 (2009).

${ }^{21}$ P. Lam, J. Wu, M. C. Tang, Q. Jiang, S. Hatch, R. Beanland, J. Wilson, R. Allison, and H. Y. Liu, Sol. Energy Mater. Sol. Cells 126, 83 (2014).

${ }^{22}$ Z. C. Xu, D. Birkedal, M. Juhl, and J. M. Hvam, Appl. Phys. Lett. 85, 3259 (2004).

${ }^{23}$ B. Sumpf, S. Deubert, G. Erbert, J. Fricke, J. P. Reithmaier, A. Forchel, R. Staske, and G. Tränkle, Electron. Lett. 39, 1655 (2003).

${ }^{24}$ J. D. Dow and D. Redfield, Phys. Rev. B 5, 594 (1972).

${ }^{25}$ D. Bimberg and N. Ledentsov, J. Phys.: Condens. Matter 15, R1063 (2003).

${ }^{26}$ M. Kolarczik, N. Owschimikow, B. Herzog, F. Buchholz, Y. I. Kaptan, and U. Woggon, Phys. Rev. B 91, 235310 (2015).

${ }^{27}$ G. Eisenstein, J. M. Wiesenfeld, M. Wegener, G. Sucha, D. S. Chemla, S. Weiss, G. Raybon, and U. Koren, Appl. Phys. Lett. 58, 158 (1991).

${ }^{28}$ L. Zhang, I. Kang, A. Bhardwaj, N. Sauer, S. Cabot, J. Jaques, and D. T. Neilson, IEEE Photonics Technol. Lett. 18, 2323 (2006).

${ }^{29}$ Y. Kaptan, H. Schmeckebier, B. Herzog, D. Arsenijević, M. Kolarczik, V. Mikhelashvili, N. Owschimikow, G. Eisenstein, D. Bimberg, and U. Woggon, Appl. Phys. Lett. 104, 261108 (2014).

${ }^{30}$ T. Vallaitis, C. Koos, R. Bonk, W. Freude, M. Laemmlin, C. Meuer, D. Bimberg, and J. Leuthold, Opt. Express 16, 170 (2008).

${ }^{31}$ J. Park, Y. D. Jang, J. S. Baek, N. J. Kim, K. J. Yee, H. Lee, D. Lee, S. H. Pyun, W. G. Jeong, and J. Kim, Opt. Express 20, 6215-6224 (2012).

${ }^{32}$ T. Akiyama, N. Hatori, Y. Nakata, H. Ebe, and M. Sugawara, Phys. Status Solidi B 238, 301-304 (2003).

${ }^{33}$ Z. C. Xu, Y. T. Zhang, A. Tackeuchi, Y. J. Horikoshi, and J. M. Hvam, Appl. Phys. Lett. 92, 063103 (2008).

${ }^{34}$ T. Switaiski, U. Woggon, D. E. A. Angeles, A. Hoffmann, J.-H. Schulze, T. D. Germann, A. Strittmatter, and U. W. Pohl, Phys. Rev. B 88, 035314 (2013).

${ }^{35}$ N. Owschimikow, M. Kolarczik, Y. I. Kaptan, N. B. Grosse, and U. Woggon, Appl. Phys. Lett. 105, 101108 (2014). 